\title{
Egyptian 2/D Table (D Composite Number): Continuation and End of a Consistent Project
}

\author{
Lionel Bréhamet \\ Independent Scholar, Bordeaux, France
}

Email address:

brehamet.1@orange.fr

\section{To cite this article:}

Lionel Bréhamet. Egyptian 2/D Table (D Composite Number): Continuation and End of a Consistent Project. History Research. Vol. 5, No. 3, 2017, pp. 16-21. doi: 10.11648/j.history.20170503.11

Received: April 11, 2017; Accepted: April 26, 2017; Published: June 21, 2017

\begin{abstract}
This final approach implies that all alternative solutions were pre-calculated by the scribes. The classification parameter is the difference (s-r) between two divisors of $\mathrm{D}$ in the decompositions $2 / \mathrm{D}=1 / \mathrm{D} 1+1 / \mathrm{D} 2$. Adequate adjustments of (s-r) provide a low limit (57) to the count of alternatives. A four-component generator $(2 / 3,2 / 5,2 / 7,2 / 11)$ operates as a (hidden) mother-table. Adding few logical rules of common sense is enough to find the reasons of the Egyptian choices. Even $2 / 95$, not decomposable into two fractions but only into three, turns out quite explainable.
\end{abstract}

Keywords: Rhind Papyrus, 2/n table, Egyptian Fractions

\section{Introduction}

A general presentation of the Egyptian $2 / D$ table and its bibliographic references was done in our first paper devoted to denominators $D$ prime [1]. A scribe named Ahmes was the transcriber of the Rhind Mathematical Papyrus (RMP), copied from an ancient copy made in the time of Amenemhat III (XII dynasty) [2]. The recto of the papyrus contains this famous table.

It should be kept in mind that the so-called ' $2 / D$ table' is not the work of only one scribe, but surely results of indeterminate periods of trials and improvements processed by a team of scribes.

In this second paper, we shall analyze only the decompositions into 2 terms if $D$ is composite.

Under a modern form we may write:

$$
2 / D=1 / \mathcal{D}_{1}+1 / \mathcal{D}_{2} .
$$

Before we start choosing decompositions for composite numbers $\mathrm{D}$, it can be assumed that in the ancient time, the results for the prime numbers were already obtained [3]. This does not necessarily mean that there was a real chronology, like 'results for D prime' before '2/D table for D composite'. These two aspects are interrelated and the project of the whole table was certainly been processed simultaneously, but in a interdependent way by a few workshops of talented scribes.

Accordingly, as it was stressed in our previous analysis [1], we have at our disposal a powerful tool, namely the four first simplest [two-terms] decompositions of $2 / \mathrm{D}$, namely $2 / 3,2 / 5,2 / 7$ and $2 / 11$.

Every composite up to $99(=3 \times 33=11 \times 9)$ may be derived from a Mother-Table looking like this:

Table 1. Mother-Table (a tool for D composite).

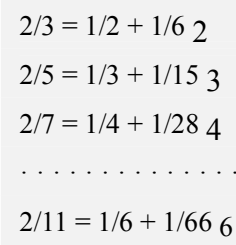

This table is far from insignificant and reveals a lot of informations, namely

$\alpha$ : Ability to have found all decompositions (unique) of $2 / \mathrm{D}$ with $\mathrm{D}$ prime

$\beta$ : Deliberate choice of stopping [2-terms] decompositions from 11 .

$\gamma$ : As maximal multiplicity of the last denominator with D is 6, keep this simple property for all other remaining decompositions with $D$ composite ${ }^{1}$.

1 As will be seen further in all tables 3,4 , and 5 , this decision is quite achievable. 
Table 2. Reordered Ahmes's selections for D composite.

\begin{tabular}{|c|c|c|}
\hline $2 / 9=1 / 6+1 / 182$ & $2 / 57=1 / 38+1 / 1142$ & \\
\hline $2 / 15=1 / 10+1 / 302$ & $2 / 63=1 / 42+1 / 1262$ & \\
\hline $2 / 21=1 / 14+1 / 422$ & $2 / 65=1 / 39+1 / 1953$ & \\
\hline $2 / 25=1 / 15+1 / 753$ & $2 / 69=1 / 46+1 / 1382$ & \\
\hline $2 / 27=1 / 18+1 / 542$ & $2 / 75=1 / 50+1 / 1502$ & \\
\hline $2 / 33=1 / 22+1 / 662$ & $2 / 77=1 / 44+1 / 3084$ & \\
\hline $2 / 35=1 / 30+1 / 42$ & $2 / 81=1 / 54+1 / 1622$ & \\
\hline $2 / 39=1 / 26+1 / 782$ & $2 / 85=1 / 51+1 / 2553$ & \\
\hline $2 / 45=1 / 30+1 / 902$ & $2 / 87=1 / 58+1 / 1742$ & \\
\hline $2 / 49=1 / 28+1 / 1964$ & $2 / 91=1 / 70+1 / 130$ & \\
\hline $2 / 51=1 / 34+1 / 1022$ & $2 / 93=1 / 62+1 / 1862$ & $2 / 95=1 / 60+1 / 3804$ \\
\hline $2 / 55=1 / 30+1 / 3306$ & $2 / 99=1 / 66+1 / 1982$ & $+1 / 5706$ \\
\hline
\end{tabular}

Our $\mathrm{m}_{2}$ numbers, multiplicity of $D_{2}$ with $D$ (when there is one), are written as subscripts.

The present study will remain faithful to our idea of a well-distributed global approach among groups of scribes. Accordingly a solution will be the result of "extensive tests applying canons of selection", so quoting the terms used in the paper of Miatello on the subject [4].

\section{All Possible Solutions (a Finite Countable Set)}

As soon the matter of composite number is approached, it becomes ineluctable to consider how the number can be decomposed in products of its divisors, as well for ancient mathematicians as for modern like us. It is possible to calculate all solutions for $D$ composite, by using a theorem little-known or ignored. We call it $N^{[2]}$ Theorem, whose we will detail some aspects. It is unlikely that these properties were known to the ancients, but an heuristic discovery is never to exclude.

Equation to be solved:

$$
N^{2}-\sigma_{1} N+\sigma_{2}=0 \text {, with integer solutions } N_{1}, N_{2} \text { and } N_{2}>N_{1} .
$$

Condition to be filled with $p$ and $q$ given:

$$
\sigma_{1} / \sigma_{2}=p / q=\text { irreducible fraction }<1 .
$$

Method: decompose $q$ in triplets of its divisors as follows

$$
q=k \times r \times s \text { with } r<s .
$$

$N^{[2]}$ Theorem: by

All the solutions are found in the finite set of $N_{1}, N_{2}$ given

$$
N_{1}=k r(r+s) / p \text { and } N_{2}=k s(r+s) / p,
$$

where $(r+s)$ is selected such that $(r+s) / p=$ integer $^{2}$.

$$
\text { Egyptian property: } 1 / N_{1}+1 / N_{2}=p / q \text {. }
$$

The relation between both solutions $N_{1}, N_{2}$ depends only on $r, s$ :

2 Of course selection due to (6) vanishes for the Egyptian table since $p=2$, and $q$, $r, \mathrm{~s}$ are odd.

$$
N_{2} / N_{l}=s / r
$$

The ratio between $N_{2}$ and $q$ is given by

$$
N_{2} / q=(r+s) / p r \text {. }
$$

If this quantity is integer, it will be labeled conventionally by $m_{2}$ (a true multiplicity), if not we will use the denomination 'fractional multiplicity' with the label $\mu_{2}$.

Another important definition will be useful, namely

$$
\Delta_{\mathrm{s}}^{\mathrm{r}}=(\mathrm{s}-\mathrm{r}) \text {. }
$$

This is the gap between two divisors of $D$ which measures the closeness between them.

Of course, the first trivial triplet decomposition of $q=1 \times$ $1 \times q$ represents nothing but a trivial identity, since the integer solutions $N_{1}=(q+1) / p$ and $N_{2}=q(q+1) / p$ satisfy

$$
1 / N_{1}+1 / N_{2}=p /(q+1)+p /[q(q+1)]=p / q .
$$

In the Egyptian $2 / q$ table $(p=2)$, if $q$ is prime, formula (11) yields the unique solution. Clearly found by the scribes. However for $q$ composite, this remains a solution among other.

This extremal solution produces the largest denominator $N_{2}$ and the highest multiplicity of $N_{2}$ with $q$, namely $m_{2}=(q$ $+1) / 2$, certainly discarded by Egyptian scribes for obvious reasons.

Furthermore there are other solutions to discard according to $\gamma$ (information of Sect. 1).

This is consistent with the last value of the Mother-table, namely 11 . Indeed, if we admit a limitation of $m_{2}$ by a topflag like

$$
\mathrm{T}_{\mathrm{f}}^{[2]}=6,
$$

then, for $p=2$, the following inequality is found:

$$
s \leq 11 r .
$$

Yes, number 11 is well the one that appears in $\beta$ (information of Sect. 1).

It has often been debated about this value 11 and the Eratosthenes's sieve as well as about the "perfectness" of the number 6, see for example Brown [5], however these notions are quite useless here. Simply, the present approach brings up an unexpected link between the numbers 11 and 6 .

Of course inequality (13) seriously reduces the number of trials for finding convenient integers $r, s$.

In our table the solutions excluded by this inequality will be displayed as barred like $z / D=1 / \mathrm{D}_{4} \neq 1 / \mathrm{D}_{z}$.

$\mathrm{Be}$ aware that the authors of the table have succeeded in calculating $2 / 35$ or $2 / 91$ where there is no multiple of $D$, then the question is not 'why these two singular cases have been chosen?' but why other existing cases have been discarded?

Since they have also certainly succeeded to calculate all possible options, we are going to list all the cases ordered according to increasing values of $\Delta_{\mathrm{s}}{ }_{\mathrm{r}}^{\mathrm{r}}$. The method is quite similar to that followed in our previous work [1], where it can be observed that all Egyptian solutions were found in the 
set $\left\{\Delta_{\mathrm{d}}, \Delta_{\mathrm{d}}, \leq 10\right\}$. The value 10 (Egyptian decimal system) obviously is not a universal constant, but seems unavoidable. Other general problems of arithmetic would probably not have benefited from such an opportunity. However once again it can be tried to limit the investigations by the following arithmetical prescription:

$$
\Delta_{\mathrm{s}}^{\mathrm{r}} \leq 10 .
$$

In Table 3, we shall gray out to indicate that an element does not satisfy this constraint, like $\Delta_{\mathrm{s}}{ }_{\mathrm{r}}$.

Condition $\Delta_{\mathrm{s}}^{\mathrm{r}} \leq 10$ is an 'absolute' condition stronger than the relative issued from (13).

Although here the divisors involved ( $s$ and $r$ ) are not those of the first denominator as in [1] but those of $D$, we were surprised that the same approach was still operating and fruitful.

Moreover, as we shall see, the "intriguing" cases $2 / 35$ and 2/91 do not need to have a separate treatment, as invoke a "arithmetic-harmonic" decomposition [5], (post) dating from ancient Greeks.

Considering such examples (and some other) as singular, or "not optimal" [6], reflects just a modern point of view, mathematically speaking. An optimality concept depends on the views, and was certainly different in ancient context.

Note: for interested readers, we have added two appendices (A and B) where we sketch out two different methods leading to the necessity of decomposing $D$ or $D^{2}$ as a product of two of its divisors.

Total cases to be analyzed (as reported in our abstract) does not take into account the cases involving a gray out or lines barred. Throughout this paper, Egyptian choices will be indicated by the abridged notation $E g$. An overview of all possibilities is displayed in Table 3 .

We see that the majority of the Egyptian choices were done according to the smallest values of $\Delta_{\mathrm{s}}{ }_{\mathrm{r}}^{\mathrm{r}}$.

Nevertheless some cases show a 'conflict' when two identical $\Delta_{\mathrm{s}}{ }^{\mathrm{r}}$ are in the presence of one-another.

As we will see below, this is not a hard problem to solve. Abdulaziz [7] is remained faithful to the spirit of ancient Egypt in substantiating its arguments with fractional quantities, then we are going to do the same by using here our 'fractional multiplicity' $\mu_{2}$, however to a lesser extent.

The closeness between 2 and the 'fractional multiplicity' $\mu_{2}$ will be estimated by a difference:

$$
\Delta_{\mu}=2-\mu_{2} \text {, (this } \Delta \text { here is written in italic). }
$$

This difference is also fractional and could not have been a source of difficulty to sort them in increasing order, for example. In this kind of work, the ancient scribes were talented!

Table 3. 2 terms decompositions with increasing values of $\Delta s^{r}$

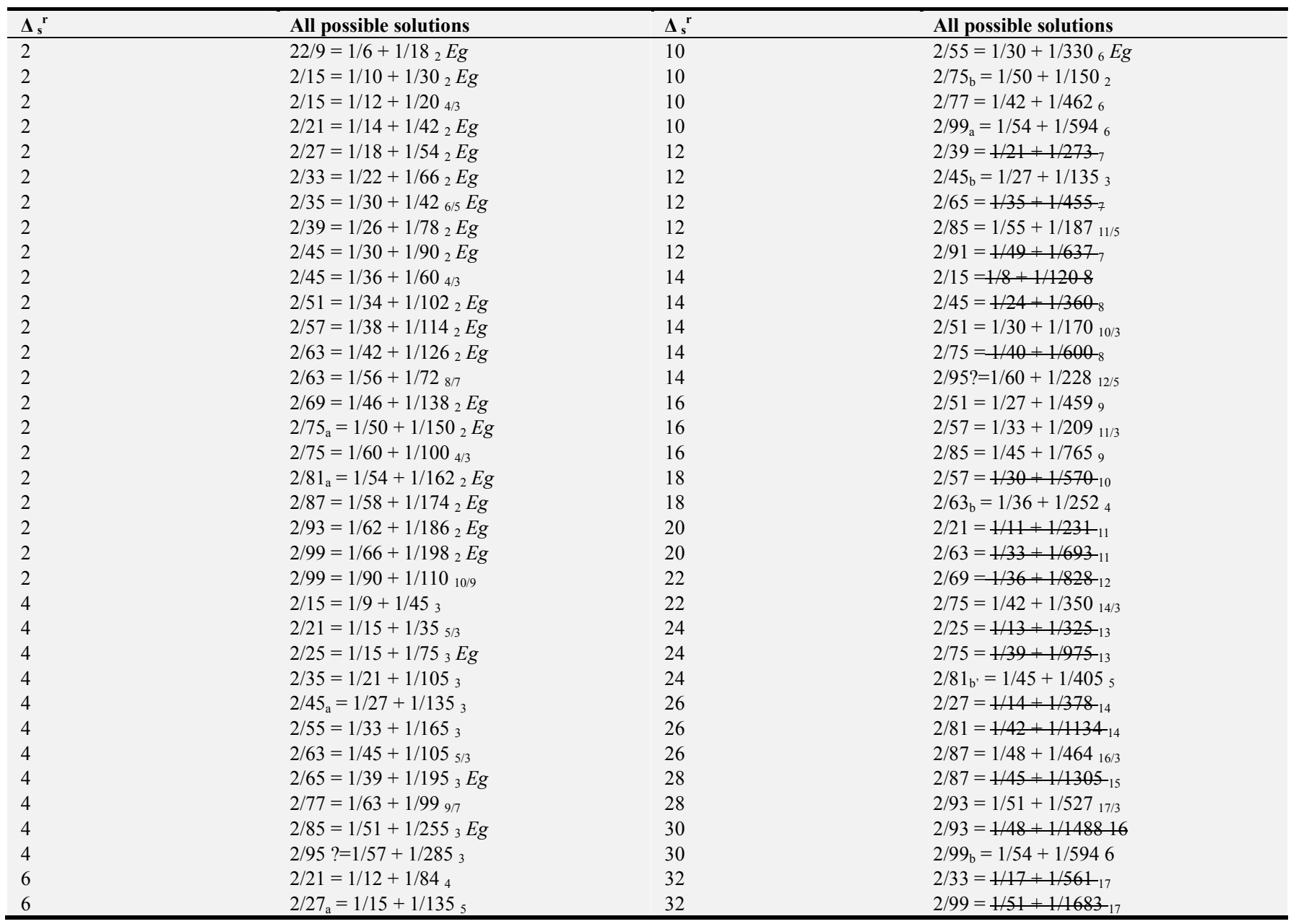




\begin{tabular}{|c|c|c|c|}
\hline$\Delta_{\mathrm{s}}{ }^{\mathrm{r}}$ & All possible solutions & $\Delta_{\mathrm{s}}{ }^{\mathrm{r}}$ & All possible solutions \\
\hline 6 & $62 / 35=1 / 20+1 / 140_{4}$ & 34 & $2 / 35=1 / 18+1 / 63 \theta_{18}$ \\
\hline 6 & $2 / 49=1 / 28+1 / 196{ }_{4} E g$ & 38 & $2 / 39=1 / 20+1 / 780_{20}$ \\
\hline 6 & $2 / 55=1 / 40+1 / 88_{8 / 5}$ & 44 & $2 / 45=1 / 23+1 / 1035_{23}$ \\
\hline 6 & $2 / 63_{a}=1 / 36+1 / 252_{4}$ & 48 & $2 / 49=1 / 25+1 / 1225-25$ \\
\hline 6 & $2 / 77=1 / 44+1 / 308_{4} E g$ & 50 & $2 / 51=1 / 26+1 / 1326-26$ \\
\hline 6 & $2 / 81_{b}=1 / 54+1 / 162_{2}$ & 54 & $2 / 55=1 / 28+1 / 1540_{28}$ \\
\hline 6 & $2 / 91=1 / 52+1 / 364_{4}$ & 56 & $2 / 57=1 / 29+1 / 1653_{-29}$ \\
\hline 6 & $2 / 91=1 / 70+1 / 130_{10 / 7} \mathrm{Eg}$ & 62 & $2 / 63=1 / 32+1 / 2016_{32}$ \\
\hline 8 & $2 / 27_{b}=1 / 15+1 / 135_{5}$ & 64 & $2 / 65=1 / 33+1 / 2145_{33}$ \\
\hline 8 & $2 / 33=1 / 21+1 / 77_{7 / 3}$ & 68 & $2 / 69=1 / 35+1 / 2415_{35}$ \\
\hline 8 & $2 / 45=1 / 25+1 / 225_{5}$ & 74 & $2 / 75=1 / 38+1 / 285 \theta_{38}$ \\
\hline 8 & $2 / 63=1 / 35+1 / 315_{5}$ & 76 & $2 / 77=1 / 39+1 / 3003_{39}$ \\
\hline 8 & $2 / 65=1 / 45+1 / 117_{9 / 5}$ & 80 & $2 / 81=1 / 41+1 / 3321_{41}$ \\
\hline 8 & $2 / 81_{a^{\prime}}=1 / 45+1 / 405_{5}$ & 84 & $2 / 85=1 / 43+1 / 365543$ \\
\hline 8 & $2 / 99=1 / 55+1 / 495_{5}$ & 86 & $2 / 87=1 / 44+1 / 382844$ \\
\hline 8 & $2 / 99=1 / 63+1 / 231_{7 / 3}$ & 90 & $2 / 91=1 / 46+1 / 418646$ \\
\hline 10 & $2 / 33=1 / 18+1 / 198_{6}$ & 92 & $2 / 93=1 / 47+1 / 4371_{47}$ \\
\hline \multirow[t]{2}{*}{10} & $2 / 39=1 / 24+1 / 104_{8 / 3}$ & 94 & $2 / 95=1 / 50+1 / 950_{10}$ \\
\hline & & 98 & $2 / 99=1 / 50+1 / 4950_{50}$ \\
\hline
\end{tabular}

Table 4. Conflicting cases with identical $\Delta{ }^{r}$.

\begin{tabular}{llll}
\hline$\Delta \mathbf{s}_{\mathbf{r}}$ & All possible solutions & Appreciations & Decisions \\
\hline 2 & $2 / 15=1 / 10+1 / 302 \mathrm{Eg}$ & $\mathrm{m}_{2}=2$ possible & $\mathrm{m}_{2}=2$ \\
2 & $2 / 15=1 / 12+1 / 204 / 3$ & $\Delta_{\mu}=42 / 63$ & \\
2 & $2 / 45=1 / 30+1 / 902 E g$ & $\mathrm{~m}_{2}=2$ possible & $\mathrm{m}_{2}=2$ \\
2 & $2 / 45=1 / 36+1 / 604 / 3$ & $\Delta_{\mu}=42 / 63$ & \\
2 & $2 / 63=1 / 42+1 / 1262 E g$ & $\mathrm{~m}_{2}=2$ possible & $\mathrm{m}_{2}=2$ \\
2 & $2 / 63=1 / 56+1 / 728 / 7$ & $\Delta_{\mu}=54 / 63$ & \\
2 & $2 / 75 \mathrm{a}=1 / 50+1 / 1502 \mathrm{Eg}$ & $\mathrm{m}_{2}=2$ possible & $\mathrm{m}_{2}=2$ \\
2 & $2 / 75=1 / 60+1 / 1004 / 3$ & $\Delta_{\mu}=42 / 63$ & \\
2 & $2 / 99=1 / 66+1 / 1982 E g$ & $\mathrm{~m}_{2}=2$ possible & $\mathrm{m}_{2}=2$ \\
2 & $2 / 99=1 / 90+1 / 11010 / 9$ & $\Delta_{\mu}=56 / 63$ & \\
6 & $2 / 91=1 / 52+1 / 3644$ & $\mathrm{~m}_{2}=4$ too far from 2 & $\mathbf{9}$ \\
non $\exists 6:$ & $2 / 91=1 / \mathrm{D} 1+1 / \mathrm{D} 22$ & virtual row: non $\exists \mathrm{m}_{2}=2$ & \\
6 & $2 / 91=1 / 70+1 / 13010 / 7 \mathrm{Eg}$ & $\Delta_{\mu}=36 / 63$ & \\
\hline
\end{tabular}

in contrast to case $2 / 81_{\mathrm{b}}=1 / 54+1 / 162$ where also $\Delta_{\mathrm{s}}{ }^{\mathrm{r}}=6$. Our second row for $2 / 91$ is virtual but useful for clarity.

Remark with Abdulaziz [7] that 2/91 is the only element that contains twice an annotation of scribe "find" on the Rhind Papyrus. Particular attention was really brought to this matter. It is not a coincidence that it appears in Table 4 . In our view, it is not comparable with $2 / 35$ which was solved immediately through its $\Delta_{\mathrm{s}}^{\mathrm{r}}=2$, by following our classification which discards $\Delta_{\mathrm{s}}{ }^{\mathrm{r}}=4,6$. Accordingly, as in our first paper [1], we stay faithful to the logic of Occam's razor: simplicity and minimal hypotheses.

Namely: if $\mathrm{m}_{2}=2$ is available, then retain it, or else adopt a value of $\mu_{2}$ closest to 2 .

Rarely a major project unfolds linearly, ie without a hitch. Nothing out of the ordinary, even today. Indeed there are still three cases unanswered, namely 2/77, 2/95 (and 2/55).

Table 5. 'no odd denominators' precept.

\begin{tabular}{ll}
\hline $\boldsymbol{\Delta}_{\mathbf{s}}{ }^{\mathbf{r}}$ & All possible solutions \\
\hline 4 & $2 / 77=1 / 63+1 / 99_{9 / 7}$ \\
6 & $2 / 77=1 / 44+1 / 308_{4} E g$ \\
10 & $2 / 77=1 / 42+1 / 462_{6}$ \\
\hline 4 & $2 / 95 ?=1 / 57+1 / 285_{3}$ \\
\hline
\end{tabular}

The 'conflicting' cases have been solved and 'forgotten'. Now another strategy must be applied in a independent way. The aim is to solve one after the other difficulties encountered during the dynamical advancement of project. Thus a 'local' decision should not interfere with those taken previously. In the present instance, a reasonable option appears to be that of the famous rule 'no odd denominators'. Only once we were forced to apply this option for solving the case 2/89 into 4 terms [1].

This was often invoked by Gillings [8], criticized by Bruins [9] and nevertheless several times used by Abdulaziz [7]. In accordance with this 'precept' (here used two times), Table 5 is displayed with some barred rows indicating what possibilities will be deleted. As will be shown further, there is no need apply this precept to $2 / 55$. There are two immediate effects: the smallest $\Delta_{\mathrm{s}}{ }^{\mathrm{r}}=6$ is chosen for $2 / 77$ and there is no more possibility for $2 / 95$ ! Thus the conclusion is that:

2/77=1/144+1/308 $4 \mathrm{Eg}$ and 2/95 has no decomposition into two terms.

Up to this point, the utilization rates of mother-table 1 with various divisors, are the following: 
Table 6. Incomplete use of Mother-Table

\begin{tabular}{llll}
\hline Mother-rows & $\begin{array}{l}\text { Number of } \\
\text { times used }\end{array}$ & Examples & Divisors \\
\hline $2 / 3=1 / 2+1 / 6_{2}$ & 16 & $2 / 51=[$ row1] $/ 17$ & 17 \\
$2 / 5=1 / 3+1 / 15_{3}$ & 3 & $2 / 65=[$ row2] $/ 13$ & 13 \\
$2 / 7=1 / 4+1 / 28_{4}$ & 2 & $2 / 77=[$ row3] $/ 11$ & 11 \\
$2 / 11=1 / 6+1 / 66_{6}$ & 0 & no $=[$ row4] $? ?$ & $? ?$ \\
\hline
\end{tabular}

In the current dynamic approach, it is the time to ask the question "what becomes $2 / 55$ ? ".

Table 7. the three last options for 2/55.

\begin{tabular}{ll}
\hline $\boldsymbol{\Delta}_{\mathbf{s}}{ }^{\mathbf{r}}$ & Remaining solutions for $\mathbf{2 / 5 5}$ \\
\hline 4 & $2 / 55=1 / 33+1 / 1653$ \\
6 & $2 / 55=1 / 40+1 / 888 / 5$ \\
10 & $2 / 55=1 / 30+1 / 3306 \mathrm{Eg}$ \\
\hline
\end{tabular}

Please note that the relation of 2/55 with the mother-table is quite unique. In our previous paper [1] we showed that there were reasons for leaving the decompositions into 2 terms from the value 11 .

This explained why start the remaining cases for $\mathrm{D}$ prime in [3- or 4-terms] from 13 (except for 23 that we have quite justified). Furthermore this fact assigned the quality of mother-table to the first four fractions. If the case $2 / 55$ does not use $\{2 / 11\}$ as template, this quality is lost, and a lot of things become un-understandable or inconsistent.

This is why scribes adopted an ultimate decision for $2 / 55$ :

Table 8. Status of 2/55 once completed the use of Mother-Table.

\begin{tabular}{llll}
\hline Ahmes's selection & Mother-row & Divisor & $\begin{array}{l}\text { Number of } \\
\text { times used }\end{array}$ \\
\hline $2 / 55=1 / 30+1 / 330_{6} E g$ & $2 / 11=1 / 6+1 / 66_{6}$ & 5 & 1 \\
\hline
\end{tabular}

Since the last case 2/95 has no solution into 2 terms, a decomposition should be found elsewhere.

A solution within easy reach is simple by observing that 95 $=5 \times 19$.

2/19 has been already [1] calculated in [3-terms] (but not $2 / 5$ ), then it is enough to divide $2 / 19$ by 5 :

$\left(2 / 19=1 / 12+1 / 76_{4}+1 / 114_{6}\right) / 5=2 / 95$

$=1 / 60+1 / 3804+1 / 5706$ Eg $($ Egyptian final result $)$.

This does not undermine the role of the [2-terms] mothertable and (what is more) agrees with the prescription of $\gamma$ sect. 1, discarding any multiplicity of the last denominator beyond of 6 .

Remark: with their experience in the [4-terms] analysis and using the same methods applied to a composite number, the scribe team could have tried to search for a solution into 4 terms. Unfortunately the least bad solution is $2 / 95=1 / 90+$ $1 / 190_{2}+1 / 285_{3}+1 / 855_{9}$. Of course rejected!

\section{Conclusion}

Yes, by discovering a key parameter like $\Delta_{\mathrm{s}}{ }_{\mathrm{r}}$, there were still improvements to be made to the most recent theories namely that of Abdulaziz [7].

As it was seen, Table 2 (2/D with $D$ composite) was easily re-constructed using a global approach.
Furthermore we deliberately used not too much arithmetical analysis because that was not needed. We never needed assumption as to favor the largest denominator $D_{l}$. Moreover, this occurrence appears only a few times (2) in our lists. Used by some authors, it is not a selection criterion in our approach. Looking back at the successive steps of selection, it could appear as incoherent for us (modern mathematicians) because we would have had the reflex to analyze all case by case and in ascending order as $2 / 9,2 / 15$, $2 / 21 \cdots$. Selections were made in the following order:

$$
\begin{gathered}
\{2 / 9,2 / 21,2 / 27,2 / 33,2 / 35,2 / 39,2 / 51,2 / 57,2 / 69,2 / 81 \\
2 / 87,2 / 93,2 / 25,2 / 65,2 / 85,2 / 49\}
\end{gathered}
$$

$\{2 / 15,2 / 45,2 / 63,2 / 75,2 / 99,2 / 91\},\{2 / 77\},\{2 / 55\}$ and $\{2 / 95\}$.

Nevertheless the choices made by the "builders" of Table 2 are fully coherent.

Actually, for composite numbers, this table exhibits no singularity. Singularities exist only for us.

All that is in favor for the fact that Egyptians have calculated all possible cases and analyzed these ones from preliminary tables for making their choices.

\section{Appendix A: A Key-equation}

Anyway, all the solutions for the [2-terms] cases could well have been discovered by a method far from our $N^{[2]}$ Theorem. The absence of a formalism with equations in Ancient Egypt does not forbid an heuristic discovery of a key equation like equality (22).

$D$ is decomposed as a product of two odd numbers $l$ and $m$ :

$$
D=l \times m, \text { with } l \leq m .
$$

Notice that this decomposition is not necessarily unique. $l, m$ may be prime numbers themselves or between them, or not. Case $l=1$ and $m=D$ also is possible.

Clearly the equation used for 2-terms and $D$ prime cannot longer be exploited under a primary form analogous to those used in our previous paper [1] for [3-terms] or [4-terms] series, namely

$$
1=D /\left(2 D_{1}\right)+d_{2} /\left(2 D_{1}\right) .
$$

It should be transformed into a form approximately similar, by naturally imagining to redistribute both factors $l, m$, each for one fraction (in the sense used by Bruins [7]) like as:

$$
l=m /\left(2 D_{1}\right)+l /\left(2 D_{1}\right),
$$

whence

$$
2 / \mathrm{D}=1 /\left(1 \mathrm{D}_{1}\right)+1 /\left(\mathrm{m} \mathrm{D}_{2}\right) .
$$

A mixed form (simultaneously additive and multiplicative) shows what is the problem to solve:

$$
2 D_{1} D_{2}=l D_{1}+m D_{2} \text {. }
$$


After a lot of trials, it depends on the intuition ability of scribes (or ourselves) for realizing that solutions $D_{1}$ could be 'centered' around $m / 2$ as well as $D_{2}$ around $l / 2$, as suggested by (18). Thus, a first idea is to set

$$
2 D_{1}=m+r_{1} \text { and } 2 D_{2}=l+s_{2},
$$

where $r_{1}, s_{2}$ obviously are unknown odd numbers to be found by avoiding $r_{1}=m$ and $s_{2}=l$ which would lead to $D_{1}=D_{2}$.

From the two above equations, it can derived a keyequation, only multiplicative, and more or less unexpected, namely

$$
r_{1} s_{2}=D(=l m) \text {. }
$$

Once a doublet $\left\{r_{1}, s_{2}\right\}$ chosen from this equation amazingly simple, all the solutions of the initial problem [see(1),(19)] are available, since (in modern algebraic notations)

$$
D_{1}=l\left(m+r_{1}\right) / 2 \text { and } D_{2}=m\left(l+s_{2}\right) / 2 \text {. }
$$

We cannot argue that the solutions were found by this way, of course.

\section{Appendix B: A Babylonian Track}

From the basic equation

$$
2 / D=1 / D_{1}+1 / D_{2},
$$

we could search for a decomposition of $2 / D$ as a sum of two other terms, each smaller than $2 / D$, such that the sum to be $2 / D$.

$$
2 / D=2 / D_{1}+2 / D_{2},
$$

ie with a $D_{1}$ not too close to $D$ by an amount of $k_{1}$ and a $D_{2}$ not too close to $D$ by an amount of $k_{2}\left(>k_{1}\right)$. Actually this yields a decomposition of 1 :

$$
1=D /\left(D+k_{1}\right)+D /\left(D+k_{2}\right) .
$$

With these definitions, (26) leads to

$$
k_{1} k_{2}=D^{2}
$$

This gives the final solutions (in modern algebraic notations)

$$
\mathcal{D}_{1}=\left(D+k_{1} /\right) / 2 \text { and } \mathcal{D}_{2}=\left(D+k_{2}\right) / 2 .
$$

Be careful to calligraphic notations for $\mathcal{D}_{1}$ and $\mathcal{D}_{2}$ !

Unknowns $k_{1}, k_{2}$ are not difficult to be found, with $k_{1}<k_{2}$ and $k_{1}, k_{2}$ odd numbers.

Equation (27) would tend to suggest rather a Babylonian similarity, due to their techniques of using tables of squares for calculate the product of two numbers by means of mathematical identities! This is evidenced on the tablet 'Plimpton 322' found in Senkereh (Larsa) in 1854 [10].

Besides, Ahmes says in the colophon at the beginning of papyrus that he was the copyist of an older papyrus, of Babylonian source [11]. Unexpectedly this would drive us to Pythagorean triples by the study of

$$
\left(k_{1}+k_{2}\right)^{2}=\left(k_{1}-k_{2}\right)^{2}+(2 D)^{2} .
$$

Results found in Appendix A or B lead to the same arithmetical conclusions as those obtained by our $\mathrm{N}^{[2]}$ Theorem. However, it is out of purpose of this article to develop here more.

\section{References}

[1] L. BREHAMET: Egyptian 2/D Table (D Prime Number): An Entirely New Analysis Consistent with the Idea of a Progressive Teamwork. History Research. Vol. 5, No. 2, pp. 17-29 (2017). See also L. BREHAMET: Remarks on the Egyptian 2/D table in favor of a global approach (D prime number), arXiv: 1403.5739 [math. HO] (2014).

[2] M. CLAGETT: Ancient Egyptian Science: A source book, American Philosophical Society, Vol. 3, p. 113 (1999).

[3] B. L. van der Waerden: "The (2:n) Table in the Rhind Papyrus". Centaurus Vol. 23, 259-74 (1980). For a probable derivation of composites from prime numbers, see pp. 265 66 .

[4] L. MIATELLO: "The Values in the Opening Section of the Rhind Mathematical Papyrus", Physis - Rivista Internazionale di Storia della Scienza Vol. 44, pp.327-347 (2007).

[5] K. BROWN: The Rhind Papyrus 2/n Table (1995), available on the site http://www.mathspages.com/home/kmath340/kmath340.htm.

[6] M. GARDNER: Egyptian fractions: Unit Fractions, Hekats and Wages - an Update (2013), available on the site of academia.edu. [Herein can be found an historic of various researches about the subject].

[7] A. ABDULAZIZ: On the Egyptian method of decomposing 2/n into unit fractions, Historia Mathematica, Vol. 35, pp.1-18 (2008).

[8] R. J. GILLINGS: Mathematics in the Time of Pharaohs, MIT Press (1972), reprinted by Dover Publications (1982).

[9] E. M. BRUINS: The part in ancient Egyptian mathematics, Centaurus, Vol. 19, pp.241-251 (1975).

[10] O. NEUGEBAUER: The Exact Sciences in Antiquity, Copenhague, Munksgaard, (ISBN 978-0486223322), 1951.

[11] T. E. PEET: The Rhind Mathematical Papyrus, British Museum 10057 and 10058, London: The University Press of Liverpool limited and Hodder - Stoughton limited (1923). 